\title{
A biocatalytic approach to a key intermediate for the synthesis of the COVID-19 experimental drug Molnupiravir
}

\author{
Ashleigh J. Burke ${ }^{[a]}$, William R. Birmingham ${ }^{[a]}$, Ying Zhuo ${ }^{[a]}$, Bruna Zucolota da Costa ${ }^{[a]}$, Rebecca \\ Crawshaw $^{[a]}$, Thomas W. Thorpe ${ }^{[a]}$, lan Rowles ${ }^{[a]}$, James Finnigan ${ }^{[b]}$, Simon J. Charnock ${ }^{[b]}$, Sarah L. \\ Lovelock ${ }^{[a]},{ }^{*}$ Nicholas J. Turner ${ }^{[a] *}$ and Anthony P. Green ${ }^{[a] *}$
}

\begin{abstract}
Herein we report the conversion of cytidine 2 to $N$-hydroxycytidine 7 catalysed by cytidine deaminase (CD). The wild-type enzyme operates efficiently at high sustrate loadings and hydroxylamine concentrations to favor $\mathrm{N}$-hydroxy-cytidine formation over uridine. Although the wild-type enzyme demonstrated good activity, we were able to further enhance the ratio of $\mathrm{N}$-hydroxycytidine to uridine produced through directed evolution of $C D$. In particular, a T123G mutation close to the active site dramatically reduces cytidine hydrolysis activity whilst preserving desired amination activty. The approach reported provides a new route to a key intermediate for the COVID-19 experimental drug Molnupiravir 1.
\end{abstract}

Molnupiravir 1 (MK-4482 formerly known as EIDD 2801) is an experimental drug currently undergoing clinical trials for the treatment of COVID-19 (Figure 1).${ }^{[1]}$ In view of the urgent need to develop therapies for treating this pandemic there has been considerable interest in the development of cost-effective synthetic routes to this API. The original synthesis of 1 required 10 steps and proceeded in $<10 \%$ overall yield. ${ }^{[2]}$ Subsequently Vasudevan et al., reported a more streamlined approach starting from either cytidine 2 or the 5-O-butyryl derivative. ${ }^{[3]}$ Steiner et al., chose to start from uridine 3 and modified the method for introduction of the $\mathrm{N}$-hydroxy unit into the nucleobase by activation via a triazolyl derivative. ${ }^{[4]}$ Very recently the Merck group disclosed an elegant multi-enzyme approach starting from D-ribose 4 in which, after lipase-mediated 5-O-acylation to give 5, the uracil base was installed using a cocktail of four different enzymes to yield 6 . Thereafter they employed a chemical procedure for conversion to $\mathbf{1}$ which required in situ silylation of the product in order to facilitate product isolation and recovery. ${ }^{[5]}$

Inspired by the previous approaches we sought to develop a different route and one which could impact the overall efficient biocatalytic preparation of $\mathbf{1}$ on scale. All previous syntheses, including the Merck route, elected to install the $N$-hydroxy unit using chemical methods. In contrast we targeted cytidine deaminase as a potential biocatalyst for the direct conversion of cytidine 2 to $N$-hydroxy-cytidine 7 under mild conditions. Since $N$ hydroxy-cytidine 7 had previously been shown by Vasudevan et al., to undergo lipase catalysed acylation at $\mathrm{C}-5-\mathrm{OH}$, we viewed this approach as a direct way to access Molnupiravir $\mathbf{1}$ from readily available starting materials.

[a] Department of Chemistry, University of Manchester, Manchester Institute of Biotechnology, 131 Princess Street, Manchester, M1 7DN, UK; [b] Prozomix Ltd, Building 4, West End Ind. Estate, Haltwhistle, UK, NE49 9HA.

E-mail: sarah.lovelock@manchester.ac.uk;

Anthony.green@manchester.ac.uk;

nicholas.turner@manchester.ac.uk
Cytidine deaminase (CD; EC 3.5.4.5) is a zinc containing enzyme which catalyses the conversion of cytidine 2 to uridine 3. ${ }^{6]}$ The enzyme is widely distributed amongst organisms and is involved in the salvage of cytidine for uridine mono-phosphate synthesis. Interestingly cytidine deaminase has been previously applied on scale for the synthesis of non-natural nucleosides and hence it appeared to be an attractive choice as a potential industrial biocatalyst. ${ }^{[7]}$ The proposed conversion of cytidine 2 to $\mathrm{N}$-hydroxy-cytidine 7 catalysed by cytidine deaminase requires the use of hydroxylamine as the nucleophile in place of water. Although we could find no precedent for this reaction in the literature, we were intrigued by a report which demonstrated that $\mathrm{N}$-hydroxy-cytidine 7 could be hydrolysed to uridine 3 by wild-type $\mathrm{CD}$, albeit at a very low rate (ca. $2 \%$ of activity compared to cytidine 2). ${ }^{[8]}$ On the basis of this observation we initiated experiments aimed at converting cytidine 2 to $\mathrm{N}$-hydroxy-cytidine 7 catalysed by CD.

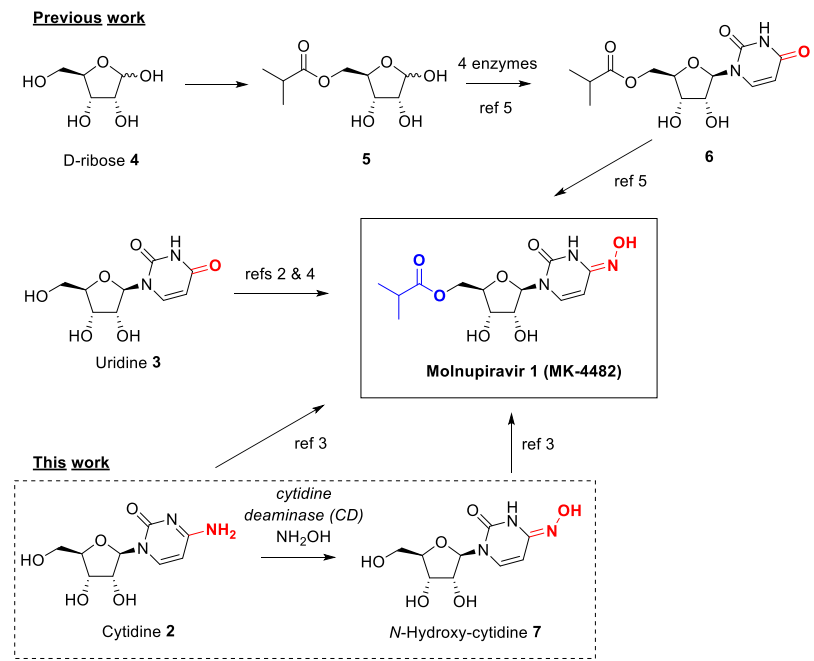

Figure 1. Different synthetic approaches to the COVID-19 experimental drug Molnupiravir 1

CD from E. coli was expressed in BL21(DE3) cells and purified to homogeneity via nickel affinity chromatography. To investigate whether this enzyme could serve as a biocatalyst for the preparation of $\mathrm{N}$-hydroxy-cytidine 7 , we initially established direct spectrophotometric assays to monitor the interconversion of cytidine 2, uridine 3 and $N$-hydroxy-cytidine 7 , based on diagnostic differences between the UV-Vis spectra of these species (Figure 2). Such assays are valuable to allow highthroughput and real-time analysis of biotransformations. In particular, the $\mathrm{N}$-hydroxy-cytidine 7 spectrum shows a diagnostic 
feature at $>300 \mathrm{~nm}$ that is not present in either cytidine 2 or uridine $\mathbf{3}$, thus allowing $N$-hydroxy-cytidine $\mathbf{7}$ formation/decay to be easily monitored at these wavelengths.

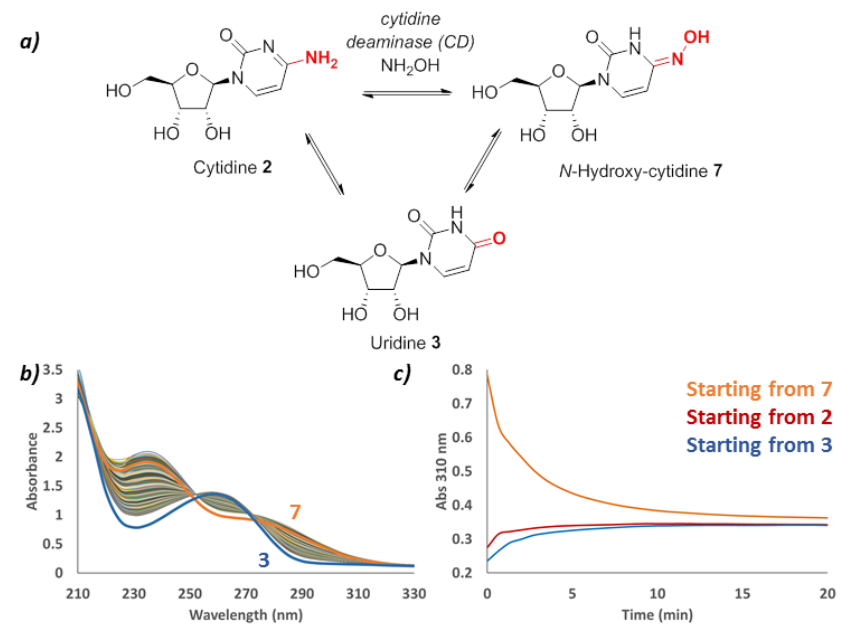

Figure 2. a) Cytidine 2, uridine $\mathbf{3}$ and $\mathrm{N}$-hydroxy-cytidine $\mathbf{7}$ can be interconverted by $\mathrm{CD}$ in the presence of $\mathrm{NH}_{2} \mathrm{OH}$. b) UV-Vis spectra showing the hydrolysis of $N$-hydroxy-cytidine $7(100 \mu \mathrm{M})$ to uridine 3 catalysed by CD (100 $\mathrm{nM})$. Spectra of authentic standards of $\mathbf{3}$ and $\mathbf{7}$ are shown in blue and orange, respectively. c) The conversion of cytidine $2(1 \mathrm{mM})$ and uridine $3(1 \mathrm{mM})$ to $N$ hydroxy-cytidine 7 by $\mathrm{CD}(5 \mu \mathrm{M})$ in the presence of $5 \% \mathrm{NH}_{2} \mathrm{OH}$ is monitored by increasing absorbance at $310 \mathrm{~nm}$. Similar final concentrations of $\mathrm{N}$-hydroxycytidine are formed using either $\mathbf{2}$ (red) or $\mathbf{3}$ (blue) as a starting material, or in reactions starting from $\mathrm{N}$-hydroxy-cytidine $\mathbf{7}$ (orange).

We initially employed this assay to investigate the conversion of $\mathrm{N}$-hydroxy-cytidine 7 to uridine 3 . Consistent with previous reports cytidine deaminase promotes $\mathrm{N}$-hydroxy-cytidine 7 hydrolysis under ambient conditions, with complete conversion of $100 \mu \mathrm{M}$ substrate achieved in 60 minutes using $100 \mathrm{nM}$ enzyme (Figure 2b). Encouraged by these results, we next turned our attention to the more challenging synthesis of $N$-hydroxy-cytidine 7 using either uridine $\mathbf{3}$ or cytidine $\mathbf{2}$ as a starting material. Pleasingly, biotransformations carried out in the presence of hydroxylamine $\left(\mathrm{NH}_{2} \mathrm{OH}\right)$ led to the rapid accumulation of a product with spectral features consistent with $\mathrm{N}$-hydroxy-cytidine 7. Similar final concentrations of $\mathrm{N}$-hydroxy-cytidine are formed using either cytidine 2 or uridine 3 as a starting material, or in reactions starting from $\mathrm{N}$-hydroxy-cytidine 7 in the presence of $\mathrm{NH}_{2} \mathrm{OH}$, suggesting that the enzyme establishes an equilibrium distribution of uridine 3 and $\mathrm{N}$-hydroxy-cytidine 7 (Figure 2c). In light of these findings, we next sought to maximize reaction productivity by increasing $\mathrm{NH}_{2} \mathrm{OH}$ and substrate loading in the biotransformations. Here we elected to use cytidine 2 as a starting material due to its reduced cost compared to uridine 3. As anticipated, increasing $\mathrm{NH}_{2} \mathrm{OH}$ concentration resulted in improved conversions to $\mathrm{N}$-hydroxycytidine 7 . Remarkably, cytidine deaminase readily tolerates high substrate loadings, $50 \% \mathrm{NH}_{2} \mathrm{OH}$ in water (as provided in bulk from commercial suppliers) as a reaction solvent, and can be lyophilized with no observable reduction in activity which is beneficial for biomanufacturing processes. Using $50 \% \mathrm{NH}_{2} \mathrm{OH}$, $200 \mathrm{mM}$ cytidine 2 is fully converted to a $4: 1$ mixture of $N$-hydroxycytidine 7 and uridine 3 within 15 minutes using $0.01 \mathrm{~mol} \%$ catalyst loading, as evidenced by HPLC analysis and analysis of the ${ }^{1} \mathrm{H}$
NMR spectrum of the crude reaction mixture. Control reactions performed under identical conditions in the absence of enzyme gave no detectable conversion to product.

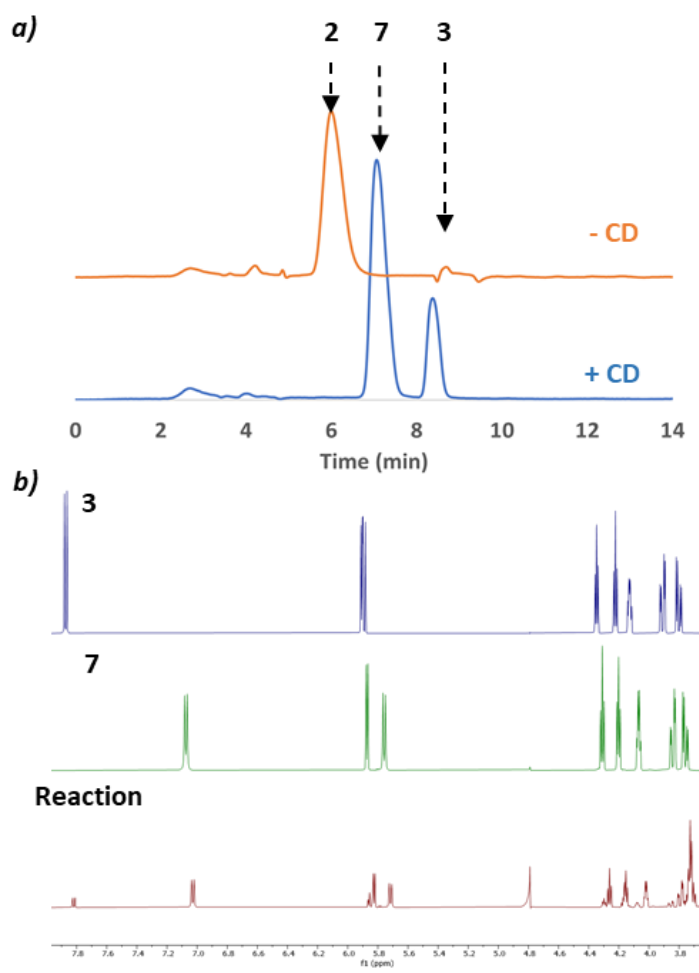

Figure 3. a) HPLC traces showing cytidine $2(200 \mathrm{mM})$ conversion to $\mathrm{N}$ hydroxy-cytidine 7 and uridine 3 by $\mathrm{CD}(20 \mu \mathrm{M})$ in the presence of $50 \% \mathrm{NH}_{2} \mathrm{OH}$ (blue). Control reactions in the absence of $\mathrm{CD}$ show no conversion (orange). Retention times of 2, 3 and $\mathbf{7}$ are identical to authentic standards. b) Stacked ${ }^{1} \mathrm{H}$ NMR traces of uridine 3 (top) $N$-hydroxy-cytidine 7 (middle) and the crude reaction mixture following the conversion of cytidine $2(200 \mathrm{mM})$ to $\mathrm{N}$-hydroxycytidine 7 and uridine 3 by $\mathrm{CD}(100 \mu \mathrm{M})$ in the presence of $50 \% \mathrm{NH}_{2} \mathrm{OH}$ (bottom). This transformation affords a $4: 1$ mixture of $7: 3$ as confirmed by HPLC analysis and analysis of the ${ }^{1} \mathrm{H}$ NMR spectra.

These results establish biotransformations with cytidine deaminase as an attractive and viable strategy for the production of $\mathrm{N}$-hydroxy-cytidine 7. Nevertheless there are clear opportunities to improve the process prior to any large scale implementation. The process currently uses high concentrations of hydroxylamine and leads to ca. $20 \%$ uridine 3 as a by-product. We anticipate that productivity could be improved through careful optimization of reaction conditions. For example, we observe that $\mathrm{N}$-hydroxy-cytidine 7 is substantially less soluble than uridine in aqueous solutions which could present an opportunity for 'in situ' product crystallization to drive the equilibrium in favour of product formation. We elected to adopt an alternative strategy, involving biocatalyst engineering via directed evolution. The aim is to reduce the natural hydrolytic activity in favour of desirable amination of cytidine by hydroxylamine to allow product accumulation under kinetic control. Similar strategies have been employed to control the partitioning of transglycosylation/hydrolysis by glycoside hydrolases. ${ }^{[9],[10]}$ To this end, we individually randomized 42 positions in the first and second coordination sphere of CD using NNK degenerate primers. 
The aforementioned spectrophotometric assay was used to evaluate individual variants as crude cell lysates arrayed in 96well plates, using $50 \mathrm{mM}$ cytidine 2 as the substrate and $1 \%$ hydroxylamine as the reaction medium. Following evaluation of 4000 clones, several point mutants (T123G, N125S, A88W) were identified which displayed the desired kinetic profiles in the spectrophotometric assay, involving rapid initial accumulation of $\mathrm{N}$-hydroxy-cytidine 7 beyond the equilibrium position followed by slower redistribution of products to equilibrium (Figure 4). This behaviour was especially pronounced in the T123G variant and was subsequently confirmed in assays with purified proteins. Reactions performed in the absence of hydroxylamine reveal that the T123G substitution leads to a dramatic reduction in the rate of cytidine hydrolysis. Biotransformations performed with $50 \%$ hydroxylamine and $200 \mathrm{mM}$ cytidine show that the T123G substitution leads to improved 6:1 ratio of $N$-hydroxy-cytidine 7 : uridine 3. Several other point mutants displaying this improved ratio were also identified during this first round of engineering. Although early in the evolutionary optimization, these data clearly demonstrate the potential to engineer cytidine deaminase to allow efficient biocatalytic production of $\mathrm{N}$-hydroxy-cytidine 7 with high yield and purity. Here the ultimate goal is to abolish hydrolytic activity whilst simultaneously improving the efficiency of cytidine amination with hydroxylamine.
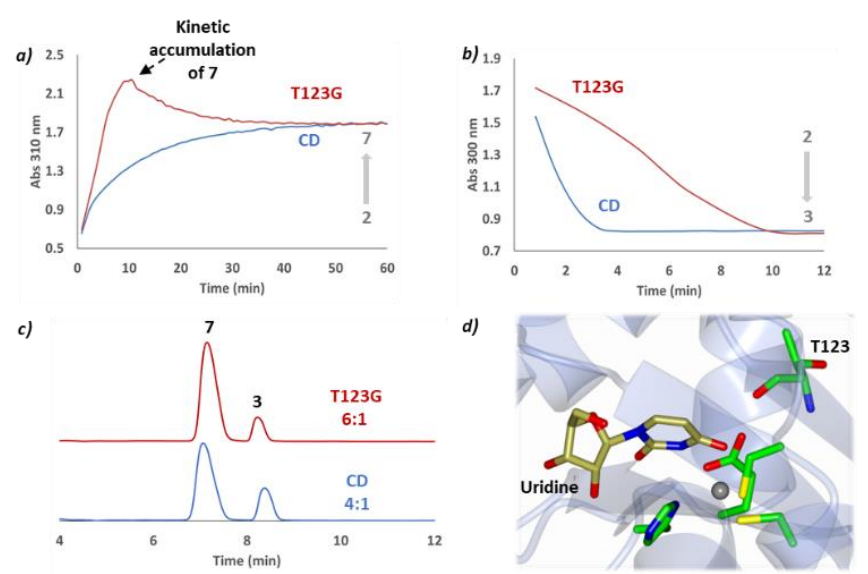

Figure 4. a) Time course for the formation of $N$-hydroxy-cytidine 7 from cytidine $2(50 \mathrm{mM})$ by wild-type CD $(2.5 \mu \mathrm{M}$, blue) and CD T123G $(2.5 \mu \mathrm{M}$, red) in the presence of $1 \% \mathrm{NH}_{2} \mathrm{OH}$. The T123G trace shows initial accumulation of $\mathrm{N}$ hydroxy-cytidine 7 beyond the equilibrium position followed by slower redistribution of products to equilibrium. b) Time course for the hydrolysis of cytidine $2(50 \mathrm{mM})$ to uridine 3 catalysed by CD $(2.5 \mu \mathrm{M}$, blue $)$ and CD T123G $(2.5 \mu \mathrm{M}$, red), showing that the T123G mutation substantially reduces cytidine hydrolysis activity. c) HPLC traces showing cytidine $2(200 \mathrm{mM})$ conversion to $N$-hydroxy-cytidine 7 and uridine 3 by CD $(20 \mu \mathrm{M}$. blue $)$ and CD T123G $(20 \mu \mathrm{M}$, red) in the presence of $50 \% \mathrm{NH}_{2} \mathrm{OH}$. The $\mathrm{T} 123 \mathrm{G}$ mutation leads to an improved $6: 1$ ratio of 7:3. d) The active site of $C D$ with uridine bound (PDB code: $1 A F 2){ }^{[11]}$ The $\mathrm{T} 123$ residue and the ligands coordinating the $\mathrm{Zn}$ cofactor are shown as atom colored sticks with green carbons.

In summary, we have developed a concise and efficient biocatalytic synthesis of $N$-hydroxy-cytidine 7 , a key intermediate for the production of Molnupiravir 1. We anticipate that further rounds of $C D$ engineering will lead to improved processes suitable for scalable manufacturing. Given that selective 5'-acylation of $\mathrm{N}$ hydroxycytidine can be achieved with lipases ${ }^{[3]}$, this work can contribute to an integrated biocatalytic strategy for Molnupiravir synthesis using cytidine as an inexpensive and readily available starting material.

\section{Acknowledgements}

We would like to thank the Bill \& Melinda Gates Foundation for funding and are particularly grateful to Dr. John Dillon, Dr. Trevor Laird and Dr. Silpa Sundaram for their helpful comments and advice during this project.

Keywords: COVID-19 cytidine deaminase $\cdot$ directed evolution - Molnupiravir.

\section{References}

[1] Cox, R. M.; Wolf, J. D.; Plemper,R. K. Nature Microbiology 2021, 6, 1118.

[2] a) Painter, G. R.; Bluemling, G. R.; Natchus, M. G.; Guthrie, D. WO2019113462, 2018. (b) Painter, G. R.; Perryman, D.; Bluemling, G. R. WO2019173602, 2019.

[3] Vasudevan, N.; Ahlqvist, G. P.; McGeough, C. P.; Paymode, D. J.; Cardoso, F. S. P.; Lucas, T.; Dietz, J.-P.; Opatz, T.; Jamison, T. F. Gupton, F. B.; Snead, D. R. Chem. Commun. 2020, 56, 13363-13364.

[4] Steiner, A.; Znidar, D.; Ötvös, S. B.; Snead, D. R.; Dallinger, D.; Kappe, C. O. ChemRxiv. 2020, DOI: 10.26434/chemrxiv.13058486.

[5] Benkovics, T.; McIntosh, J.; Silverman, S.; Kong, J.; Maligres, P.; Itoh, T.; Yang, H.; Huffman, M.; Verma, D.; Pan, W.; Ho, H.-I.; Vroom, J.; Knight, A.; Hurtak, A.; Morris, W.; Strotman, N.; Murphy, G.; Maloney, K.; Fier, P. ChemRxiv. 2020, DOI: 10.26434/chemrxiv. 13472373.v1.

[6] https://en.wikipedia.org/wiki/Cytidine_deaminase.

[7] Ferrero, M.; Gotor, V. Chem. Rev. 2000, 100, 12, 4319-4348.

[8] Trimble, R. B.; Maley, F. J. Bacteriol. 1971, 108, 145-153.

[9] Romero-Tellez, S.; Lluch, J. M.; Gonzalez-Lafont, A.; Masgrau, L. Front. Chem. 2019, 7, 200.

[10] Moulis, C.; Guieysse, D.; Morel, S.; Severac, E.; Remaud-Simeon, M. Curr. Opin. Chem. Biol. 2021, 61, 96-106.

[11] Xiang, S.; Short, S. A.; Wolfenden, R.; Carter Jr, C. W. Biochemistry 1997, 36, 4768-4774 
